\title{
Development of a Unified Numerical Kinetic Approach, Taking into Account Many-Particle Interactions in Liquid-Vapor Systems
}

\author{
Irina Shishkova*, Alexei Kryukov \\ Low Temperature Department, National Research University Moscow Power Engineering Institute, Moscow, Russia
}

Email address:

in-sh@yandex.ru (I. Shishkova), kryukovap@mail.ru (A. Kryukov)

${ }^{*}$ Corresponding author

\section{To cite this article:}

Irina Shishkova, Alexei Kryukov. Development of a Unified Numerical Kinetic Approach, taking into account Many-Particle Interactions in Liquid-Vapor Systems. American Journal of Physics and Applications. Vol. 9, No. 5, 2021, pp. 116-120. doi: 10.11648/j.ajpa.20210905.13

Received: September 4, 2021; Accepted: September 24, 2021; Published: September 30, 2021

\begin{abstract}
The study of evaporation and condensation should include consideration of heat and mass transfer processes inside the liquid, in the inter-phase transition domain, in the Knudsen layer, and in the outer area. Possible way to realize it is to use the conjugate approach, in which the description of these regions is carried out employing a single computational method. This method allows us to consider the condensed phase and gas as a single system and use the solution of kinetic equations throughout the region. Currently, processes in the gas phase have been studied quite well. The greatest obstacle to the use of kinetic equations in the condensed phase is the description of collisions involving multiple particles at the same time. In this paper a procedure is proposed to take the multi-particulate interactions within the condensed phase into account. Such approach is applied to the test study of the thermal conductivity problem for argon, neon, xenon, and krypton. Values of thermal conductivity coefficients for different quantities of interacting particles have been obtained. The comparison with corresponding experimental data is presented. Thus, the integral of paired collisions in the Boltzmann kinetic equation can be replaced by the proposed computational procedure. This approach provides a description of both liquid and gas at the level of the distribution function and ensures that the conditions at the interface are set correctly.
\end{abstract}

Keywords: Boltzmann Equation, Interface Phenomena, Joint Solution

\section{Introduction}

It is well known at present that the proper investigation of nonequilibrium processes on a vapor-condensate interface can be made based on molecular kinetic theory (MKT). The primary function of this theory is the velocity distribution function (DF) $f$ of molecules. In order to find this function, the Boltzmann kinetic equation (BKE) should be solved, which calls for the formulating boundary condition. These conditions require the knowledge of the DF for the molecules that escape from condensed matter. This DF is, as a rule, specified using various models (e.g., diffuse or mirror reflection). Less often, it is found experimentally or by setting the condensation or accommodation coefficients.

Few works have modeled liquid and gas phases within a single computational method [1-5]. Therefore, there is a need for a single framework that models both the liquid and vapor phases. One of the features of the present problem is the presence of heat and mass transfer, as seen in both liquid and vapor. During this transfer, the processes in one phase can strongly influence the phenomena in the other. Generally, the heat falling to the surface is spent on heating, and the evaporation of the liquid. At this vapor formed as a result of this evaporation is removed from the surface. The formation of a vapor flux occurs in the layer near the vapor-liquid interface. The thickness of this layer is equal to several mean free paths of vapor molecules.

Thus, the study of evaporation should include consideration of heat and mass transfer processes inside the liquid, in the interphase transition layer, in the Knudsen layer, and in the outer area. One possible way includes using the conjugate approach, in which the description of the liquid phase, interphase layer, and Knudsen layer is carried out employing a single computational method. The development 
of this approach was initiated by the authors in the paper [6]. In this paper, a model of the interaction of many particles in the condensed phase is proposed, which assumes that collisions are pair-wise independent.

The collision integral is the most complex portion of the kinetic equation, which should take into account the interaction in the condensed phase between several $(M)$ particles. According to the following research $[7,8]$, the correlations are maximal at $M=3$ and decrease rapidly with growth $M$ due to molecular chaos. Based on this fact, an approach that allows us to consider the multi-particle interaction as a set of paired collisions has been proposed [9].

This step can be considered the first approximation in the construction of a single, conjugate, end-to-end method. However, the role of correlations in the interaction of condensed matter particles among themselves is not fully understood at present. Therefore, for the further development of the conjugate approach, it is necessary to develop a new method in which the interacting particles will be considered as a single system. In this paper a version of such method is presented.

\section{Description of the Method}

\subsection{Kinetic Equation for Liquid Phase}

The presented paper proposes to solve the kinetic equation inside the condensed phase:

$$
\frac{\partial f}{\partial t}+\xi \frac{\partial f}{\partial \mathrm{r}}=Z_{M}
$$

The direct numerical solution of this type kinetic equation by the manner of $[6,9-11]$ is used.

Regarding the description of the condensed phase, the left side of equation (1) only describes the transfer of the distribution function and not the transformation of this function during the atom interactions. The value $Z_{M}$ on the right side is an analog of the paired integral of collisions but is designed to take into account the simultaneous interaction of the $M$ atoms (molecules) of the condensed phase. It describes collisions, provided that mass, momentum, and kinetic energy are preserved during the interaction, as well as the integral for paired collisions in the Boltzmann kinetic equation. In this work, $Z_{M}$ is replaced by the computational procedure described below. This method allows the use of a single numerical algorithm to determine macro parameters inside both the vapor and the liquid.

\subsection{Turn Method for Describing Multi-particle Interactions}

At both low and room temperatures, it can be assumed that each liquid particle has three degrees of freedom associated with the translational motion in Cartesian space $\mathrm{r}(x, y, z)$. A group of $M$ particles in the liquid is isolated, which were located in close proximity to each other. It is assumed that the moving particle has three degrees of freedom. Therefore, the system of $M$ particles has $N=3 M$ degrees of freedom. When passing through the equilibrium position of each particle, its velocity $\xi\left(\xi_{x}, \xi_{y}, \xi_{z}\right)$ can be fixed. Further, when constructing the computational algorithm, we will assume that the components of vector $\mathrm{P}\left(p_{1}, p_{2}, \ldots p_{j}, \ldots p_{N}\right)$ will comprise all of the velocities of atoms (molecules) before the interaction $\xi\left(\xi_{x}, \xi_{y}, \xi_{z}\right)$ :

$$
\mathrm{P}=\left[\begin{array}{l}
p_{1} \\
p_{2} \\
p_{3} \\
\cdots . . \\
p_{N-2} \\
p_{N-1} \\
p_{N}
\end{array}\right]=\left[\begin{array}{l}
\xi_{x 1} \\
\xi_{y 1} \\
\xi_{z 1} \\
\ldots . . \\
\xi_{x N} \\
\xi_{y N} \\
\xi_{z N}
\end{array}\right],|\mathrm{P}|=\sqrt{\xi_{x 1}^{2}+\xi_{y 1}^{2}+\xi_{z 1}^{2}+\ldots \ldots+\xi_{x N}^{2}+\xi_{y N}^{2}+\xi_{z N}^{2}} .
$$

Interaction between all $M$ particles will lead to a change in the state of the system, which can be described by the new vector Q. A transition of the system from one state to another as a result of the interaction of particles will be described by some operator $\mathrm{F}: \mathrm{Q}=\mathrm{FP}$. The components of the vector $\mathrm{Q}\left(q_{1}, q_{2}, \ldots q_{j}, \ldots q_{N}\right)$ will then be the velocities of the condensed phase particles after the interaction $\xi^{\prime}\left(\xi_{x}^{\prime}, \xi_{y}^{\prime}, \xi_{z}^{\prime}\right)$ :

$$
\mathrm{Q}=\left[\begin{array}{l}
q_{1} \\
q_{2} \\
q_{3} \\
\cdots . . \\
q_{N-2} \\
q_{N-1} \\
q_{N}
\end{array}\right]=\left[\begin{array}{l}
\xi_{x 1}^{\prime} \\
\xi_{y 1}^{\prime} \\
\xi_{z 1}^{\prime} \\
\ldots . . \\
\xi_{x N}^{\prime} \\
\xi_{y N}^{\prime} \\
\xi_{z N}^{\prime}
\end{array}\right],|\mathrm{Q}|=\sqrt{\xi_{x 1}^{\prime 2}+\xi_{y 1}^{\prime 2}+\xi_{z 1}^{\prime 2}+\ldots \ldots+\xi_{x N}^{\prime 2}+\xi_{y N}^{\prime 2}+\xi_{z N}^{\prime 2}}
$$


The matrix A of size $N$ by $N(N=3 M)$ can correspond to operator $\mathrm{F}$, and the transformation of the original state vector can be described by the matrix product $\mathrm{Q}=\mathrm{AP}$. The last transformation can be represented as a system of linear equations:

$$
\begin{aligned}
& a_{11} p_{1}+a_{12} p_{2}+\ldots+a_{1 j} p_{j}+\ldots+a_{1 N} p_{N}=q_{1} \\
& a_{21} p_{1}+a_{22} p_{2}+\ldots+a_{2 j} p_{j}+\ldots+a_{2 N} p_{N}=q_{2} \\
& \left.a_{i 1} p_{1}+a_{i 2} p_{2}+\ldots+a_{i j} p_{j}+\ldots+a_{i N} p_{N}=\quad q_{i}\right\} \text {, } \\
& a_{N 1} p_{1}+a_{N 2} p_{2}+\ldots+a_{N j} p_{j}+\ldots+a_{N N} p_{N}=q_{N}
\end{aligned}
$$

where $a_{i j}$ are elements of matrix $\mathrm{A}$.

In the numerical implementation of the proposed approach, the principal point is the finding of elements of the transformation matrix.

Enter the following assumptions:

1. The norm of vector $\mathrm{Q}$ is equal to the norm of vector $\mathrm{P}$.

2. The motion of the condensed phase atoms (molecules) is chaotic.

3. All collisions between particles occur simultaneously.

The first condition signifies the observance of the law of energy conservation during the interactions of the condensed phase particles. Condition 2 ensures linear independence of all equations included in the system (4).

Furthermore, conditions 2 and 3 at this stage of research allow us to interpret the process of multi-particle interaction as the rotation of a vector $\mathrm{P}$ in multivariate space $\left(e_{1}, e_{2}, e_{3}, \ldots, e_{k}, \ldots, e_{l}, \ldots, e_{N}\right)$ with dimension $N=3 M$. A similar idea was previously implemented by one of the authors in work [12] to take into account the internal degrees of freedom of molecules of a complex structure in gas (vapor).

Each degree of freedom will have its own coordinate axis. For example, $e_{1} \leftrightarrow \xi_{x 1}, e_{2} \leftrightarrow \xi_{y 1}, e_{3} \leftrightarrow \xi_{z 1}$. In this example, $\mathrm{F}$ is the turn operator. It is necessary to construct a turn matrix $\mathrm{A}$ in the space of dimension $3 M$ to mathematically describe the transformation $\mathrm{Q}=\mathrm{FP}$. The desired vector $\mathrm{Q}$ is then calculated as the product of the turn matrix $\mathbf{A}$ by the original vector $\mathrm{P}: \mathrm{Q}=\mathrm{AP}$. The turn angle is assumed to be randomly selected.

Since turn in $\mathrm{N}$-dimensional space is considered as a sequence of turns in planes $\left(e_{k}, e_{l}\right)$, a matrix $\mathrm{A}$ can be found as a sequential product of all matrices $\mathrm{A}_{k l}$ :

$$
\mathrm{A}=\mathrm{A}_{12} \mathrm{~A}_{13} \mathrm{~A}_{23} \ldots \mathrm{A}_{k l} \ldots
$$

After finding the vector $\mathrm{Q}$, all components of the particle velocities after the interaction are known. The proposed approach plans to create the possibility to find the distribution function directly from the computational procedure itself without resorting to recording a complex multi-particle collision integral.

\section{Testing the Method by Solving of Thermal Conductivity Problem}

\subsection{Statement of the Thermal Conductivity Problem}

The statement of the problem is presented in Figure 1. A thin layer of the specified substance with thickness $L$ is limited by impermeable surfaces that have temperatures $T_{1}$ and $T_{2}$. The problem was solved for two cases: $T_{1} / T_{2}=1.1$ and $T_{1} / T_{2}=1.001$.

The lower temperature $T_{2}$ is taken as the base $T_{0}=T_{2}$, and the length of the region is taken as $L \approx(300-500) d$. Here $d$ is the diameter of the molecule of the selected substance.

The occurrence of heat flux at heating the left surface with respect to the right is interpreted as follows: as the temperature increases, the kinetic energy of atoms and the amplitude of their oscillations near the bottom of the potential well also increase. As a result of the interactions of neighboring atoms, energy is transferred from the left to the right (i.e., heat transfer is carried out). According to the condition of the problem, the temperatures $T_{1}$ and $T_{2}$ are kept constant. Thus, at a stationary statement, the heat is continuously supplied to the left, and the same amount is withdrawn from the right.

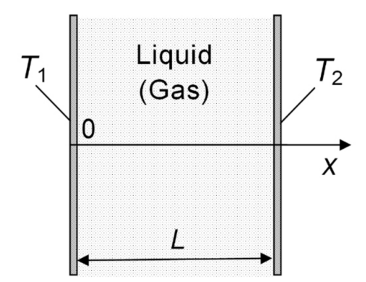

Figure 1. Statement of the thermal conductivity problem.

\subsection{Mathematical Model}

The kinetic energy of the total condensate volume under consideration remains the same as the basic conversion $\mathrm{Q}=\mathrm{FP}$ of the present work described above. It should be noted that this constancy of kinetic energy in the transition from the condensed phase to the vapor layer (in the so-called interphase) was demonstrated by molecular dynamics (MD) methods in the article [13].

The mathematical model expressed in formulas (1-5) explicitly includes only the kinetic energy of the particle motion. However, potential energy is present in the very idea of the turn method, as the proposed model assumes that many particles are connected to each other and interact simultaneously. This situation is only possible if there is a potential field in which all particles are located, and potential energy plays a key role in energy exchange, although it does not change the total kinetic energy or the length of the state vectors $\mathrm{P}$ or $\mathrm{Q}$.

Kinetic equations (1) were solved in order to determine the thermal conductivity coefficients for different substances at 
various temperatures. The heat flux in the direction $x$ was defined as the third-order moment of the distribution function:

$$
q_{x}=\frac{1}{2} m \int(\xi-\mathrm{u})^{2}\left(\xi_{x}-u_{x}\right) f d \xi
$$

where $d \xi=d \xi_{x} d \xi_{y} d \xi_{z}, \mathrm{u}\left(u_{x}, u_{y}, u_{z}\right)$ is the mean particle velocity vector, $k$ is the Boltzmann constant, $m$ is the mass of the molecule.

Then, thermal conductivity coefficients were calculated by the formula:

$$
\lambda=q_{x} L /\left(T_{1}-T_{2}\right) .
$$

To solve the problem of heat flow in the liquid, the Chapman-Enskog function [14] was taken as boundary conditions:

$$
f_{1}=f_{M}\left[1-\frac{q_{x}}{p} \frac{c_{x}}{R T}\left(1-\frac{1}{5} \frac{\mathrm{c}^{2}}{R T}\right)\right]
$$

where $f_{M}$ is the Maxwell distribution function calculated at temperature $T=T_{1}$ or $T=T_{2}$, and density was determined from the condition of impermeability of surfaces; $c_{x}=\xi_{x}-u_{x} ; \mathrm{c}^{2}=(\xi-\mathrm{u})^{2} ; p-$ pressure.

Substituting values $q_{x}$ in formula (6), as well as boundary temperatures $T_{1}$ or $T_{2}$ and solving equations (1) with Chapman-Enskog functions as the boundary conditions, we will obtain values of heat flow and temperature difference. Initially, they may not coincide with those substituted in (8). Therefore, an iterative process is needed until an agreement between $T_{1}, T_{2}$, and $q_{x}$ is reached in both the formula and the results. Next, the thermal conductivity coefficient in the liquid is determined in accordance with (7).

\subsection{Results and Analysis}

Examples of the results of the calculation of thermal conductivity coefficients for different substances are

\begin{tabular}{|c|c|c|c|c|c|c|c|}
\hline$T_{0}, \mathbf{K}$ & $M$ & $\lambda \times 10^{3}, \mathrm{~W} / \mathrm{mK}$ & $\lambda_{\text {tabl }} \times 10^{3}, \mathrm{~W} / \mathrm{mK}$ & $T_{0}, \mathbf{K}$ & $M$ & $\lambda \times 10^{3}, \mathrm{~W} / \mathrm{mK}$ & $\lambda_{\text {tabl }} \times 10^{3}, \mathrm{~W} / \mathrm{mK}$ \\
\hline \multirow[t]{2}{*}{ Argon } & & & & Neon & & & \\
\hline & 3 & 134.1 & & & 3 & 145.7 & \\
\hline \multirow[t]{2}{*}{ 87. $(\mathrm{p}=0.1 \mathrm{MPa})$} & 4 & 116.4 & 130. & $25 .(p=0.05 \mathrm{MPa})$ & 4 & 126.5 & 117. \\
\hline & 5 & 93.57 & & & 5 & 101.7 & \\
\hline \multirow{2}{*}{ 100. $(p=1 \mathrm{MPa})$} & 4 & 124.8 & \multirow{2}{*}{110.9} & \multirow{2}{*}{$27 .(p=0.1 \mathrm{MPa})$} & 4 & 131.5 & \multirow{2}{*}{113.} \\
\hline & 5 & 100.3 & & & 5 & 105.7 & \\
\hline 120. $(p=2 \mathrm{MPa})$ & 5 & 109.9 & 85.5 & 30. $(p=0.22 \mathrm{MPa})$ & 5 & 111.4 & 92. \\
\hline \multirow{3}{*}{ 130. $(\mathrm{p}=3 \mathrm{MPa})$} & 5 & 114.4 & \multirow{3}{*}{73.6} & \multirow{3}{*}{ 103. $(p=0.1 \mathrm{MPa})$} & 2 & 28.95 & \multirow{3}{*}{22.7} \\
\hline & 6 & 78.46 & & & 3 & 23.92 & \\
\hline & 7 & 62.77 & & & 4 & 20.14 & \\
\hline \multirow{2}{*}{$200 .(p=0.1 \mathrm{MPa})$} & 2 & 13.84 & \multirow{2}{*}{12.6} & \multirow{2}{*}{ 198. $(\mathrm{p}=0.1 \mathrm{MPa})$} & 2 & 40.14 & \multirow{2}{*}{37.1} \\
\hline & 3 & 10.81 & & & 3 & 33.16 & \\
\hline \multirow{2}{*}{ 300. $(p=0.1 \mathrm{MPa})$} & 2 & 16.95 & \multirow{2}{*}{17.7} & \multirow{2}{*}{ 273. $(p=0.1 \mathrm{MPa})$} & 2 & 47.14 & \multirow{2}{*}{46.1} \\
\hline & 3 & 13.24 & & & 3 & 38.94 & \\
\hline Krypton & 3 & 97.35 & \multirow{3}{*}{87.6} & \multirow{3}{*}{ 170. $(\mathrm{p}=0.13 \mathrm{MPa})$} & 3 & 73.94 & \multirow{3}{*}{64.0} \\
\hline \multirow[t]{2}{*}{ 125. $(\mathrm{p}=0.14 \mathrm{MPa})$} & 4 & 84.51 & & & 4 & 64.21 & \\
\hline & 5 & 67.94 & & & 5 & 51.62 & \\
\hline \multirow{3}{*}{ 130. $(p=0.2 \mathrm{MPa})$} & 3 & 99.28 & \multirow{3}{*}{84.0} & \multirow{3}{*}{ 180. $(\mathrm{p}=0.22 \mathrm{MPa})$} & 3 & 76.11 & \multirow{3}{*}{65.5} \\
\hline & 4 & 86.19 & & & 4 & 66.07 & \\
\hline & 5 & 69.29 & & & 5 & 53.11 & \\
\hline & 2 & 6.96 & & & 2 & 4.96 & \\
\hline 180. $(\mathrm{p}=0.1 \mathrm{MPa})$ & 3 & 5.22 & 5.98 & $250 .(\mathrm{p}=0.1 \mathrm{MPa})$ & 3 & 3.63 & 4.76 \\
\hline & 2 & 8.99 & 9.58 & $300(\mathrm{p}=0.1 \mathrm{MPa})$ & 2 & 5.44 & 5.69 \\
\hline $300 .(p=0.1 \mathrm{MPa})$ & 3 & 6.74 & 9.58 & $300(\mathrm{p}=0.1 \mathrm{MPa})$ & 3 & 3.97 & 5.09 \\
\hline
\end{tabular}
presented in Table 1.

Table 1. Thermal conductivity factors for $\mathrm{Ar}, \mathrm{Ne}, \mathrm{Kr}$, and Xe.

The last columns of all tables contain values taken from reference literature [15].

It is important to note that the nonequilibrium ChapmanEnskog boundary functions for liquid and half- Maxwell functions at diffuse reflection scheme for gas were used for obtaining the data in the table.

As observed from the results obtained, the experimental data $\left(\lambda_{t a b l}\right)$ are consistent with the calculation results at $M=3-5$ for liquids and at $M=2-3$ for gases with different densities. Table 1 also demonstrates that at high enough pressures, the number of interacting argon particles tends to be $5-7$. The quantity of particles able to interact at normal conditions for all gases $M$ is close to 2 .

The results presented were obtained for the length of the calculating region $L \approx(300-500) d$. Obviously, the temperature difference $T_{1} / T_{2}=1.1$ is too large for such a thin liquid layer. Therefore, it was important to make calculations using the proposed approach for the lowest temperature ratio.

Figure 2 shows the dependence dimensionless heat flux on 
$x$ at $T_{1} / T_{2}=1.001$ and the number of interacting particles $M=4$.

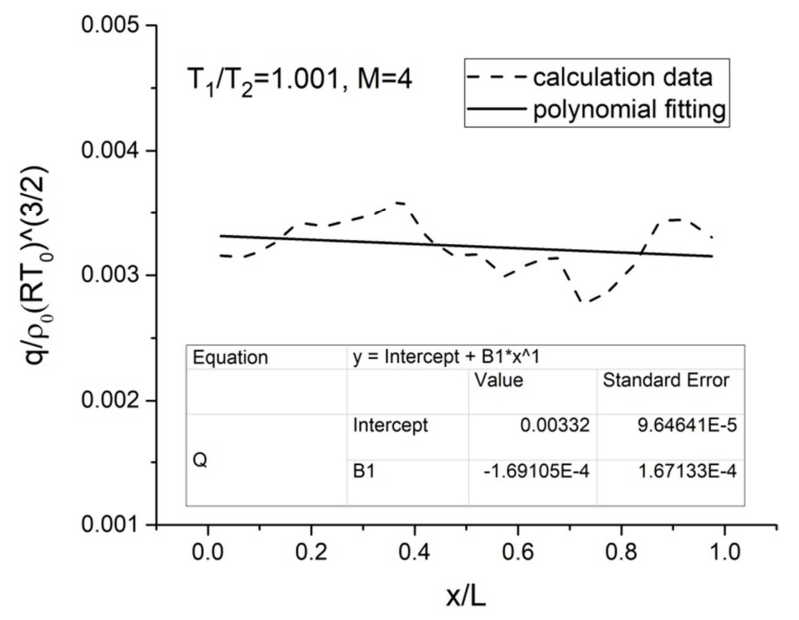

Figure 2. Dependence of heat flux density on $x$ at a low temperature ratio.

for limiting surfaces. $\rho_{0}$ is density at $T_{0}$ and $p, R$ is individual gas constant, for large enough $T_{0} \quad \rho_{0}=\mathrm{p} / R T_{0}$.

The heat flux is shown by a dashed line. A solid line indicates the result of polynomial interpolation using Origin 9. Thus, the spread of the computed data is statistically processing, and the resulting solid line is physically correct.

Further reduction of the temperature ratio does not allow us to obtain adequate results. Thus, $T_{1} / T_{2}=1.001$ is the limit for the proposed approach at this stage of the work.

\section{Conclusion}

An approach has been proposed to take the multiparticulate interactions within the condensed phase into account. At the first stage this approach was applied to the study of the thermal conductivity problem for argon, neon, xenon, and krypton. Values of thermal conductivity coefficients for different numbers of interacting particles were obtained. Satisfactory agreement was found with the experimental data at $M=3-5$ for liquid and $M \approx 2$ for gases under normal conditions.

This approach is planned to apply in close future for evaporation-condensation problems solving without using of any empirical values concerning interface: accommodation coefficients and so on. In evaporation-condensation processes substance transfers from liquid (solid) to vapor. The velocity distribution functions of molecules for condensate and vapor can be obtain by suggested method as solution result. Then any macroscopic values such as density, temperature, mass, momentum, heat fluxes are determined as integrals of distribution functions in $3 \mathrm{D}$ velocity space.

\section{Acknowledgements}

The work was carried out with the support of the Russian Foundation for Basic Research (project No. 2008-00342).

\section{References}

[1] A. Frezzotti and P. Barbante. Simulation of shock induced vapor condensation flows in the Lennard-Jones fluid by microscopic and continuum models. Phys. Fluids 32, 2020, 122106.

[2] P. Barbante and A. Frezzotti. A comparison of models for the evaporation of the Lennard-Jones fluid. Eur. J. Mech.: B/Fluids 64, 2017, pp. 69-80.

[3] M. Kon, K. Kobayashi, and M. Watanabe. Method of determining kinetic boundary conditions in net evaporation/condensation. Phys. Fluids 26, 2014, 072003.

[4] M. Kon, K. Kobayashi, M. Watanabe, Liquid temperature dependence of kinetic boundary condition at vapor-liquid interface. Int. J. Heat Mass Transfer 99, 2016, pp. 317-326.

[5] S. Busuioc, L. Gibelli, D. A. Lockerby, and J. E. Sprittles. Velocity distribution function on spontaneously evaporating atoms. Phys. Rev. Fluids 5, 2020, 103401.

[6] Shishkova I. N., Kryukov A. P., Levashov V. Yu. Study of evaporation-condensation problems: from liquid through interface surface to vapor. International Journal of Heat and Mass Transfer. V. 112, 2017, pp. 926-932.

[7] V. Ya. Rudyak. Statistical theory of dissipative processes in gases and liquids. Novosibirsk. 1987 (in Russian).

[8] A. A. Tsykalo, M. M. Kontsov. Study of the effect of threeparticle non-additive interactions on the thermodynamic properties of dense gases and liquids. Journal of Technical Physics. T. 47, No. 12, 1977, pp. 2601-2607 (in Russian).

[9] Shishkova I. N., Kryukov A. P., Levashov V. Y. Vapour-liquid jointed solution for the evaporation-condensation problem. International Journal of Heat and Mass Transfer. Vol. 141, 2019, pp. 9-19.

[10] I. N. Shishkova, A. P. Kryukov and V. Yu. Levashov. Joint liquid-vapor approach development at solution of different heat and mass transfer problems. Journal of Physics: Conference Series. ICFEPT 2019, 2019, 1370: 012019. doi: $10.1088 / 1742-6596 / 1370 / 1 / 012019$

[11] F. G. Tcheremissine, Discrete approximation and examples of solutions of the Boltzmann equation, in: Computational Dynamics of Rarefied Gas, Computer Center of the Russian Academy of Sciences, 2000, pp. 37-74.

[12] I. N. Shishkova, S. S. Sazhin, J. -F. Xie. A solution of the Boltzmann equation in the presence of inelastic collisions. $J$. Computational Physics. V. 232, 2013, pp. 87-99.

[13] V. V. Zhakhovsky, A. P. Kryukov, V. Yu. Levashov, I. N. Shishkova, S. I. Anisimov Mass and heat transfer between evaporation and condensation surfaces: Atomic simulation and solution of Boltzmann kinetic equation. Proceedings of the National Academy of Sciences, PNAS April 16, 2018. 201714503; published ahead of print April 16. https://doi.org/10.1073/pnas. 1714503115 or Proc. Nat. Acad. Sci. 116 18209, 2019.

[14] M. N. Kogan, Rarefied Gas Dynamics, Plenum, New York, 1969.

[15] NIST-JANAF Thermochemical Tables. https://janaf.nist.gov/. 